\title{
Routinedaten aus Notaufnahmen: Unterschiedliche Dokumentationsanforderungen, Abrechnungsmodalitäten und Datenhalter bei identischem Ort der Leistungserbringung
} Routine Data from Emergency Departments: Varying Documentation Standards, Billing Modalities and Data Custodians at an Identical Unit of Care

\section{(우(1) $(9)$}

Autoren

Felix Greiner ${ }^{1}$, Anna Slagman², 3, Christoph Stallmann4, Stefanie March4, Johannes Pollmanns ${ }^{5}$, Patrik Dröge ${ }^{6}$, Christian Günster ${ }^{6}$, Marie-Luise Rosenbusch7, Joachim Heuer ${ }^{7}$, Saskia E. Drösler ${ }^{5}$, Felix Walcher ${ }^{1}$, Dominik Brammen ${ }^{1,8}$

Institute

1 Medizinische Fakultät, Universitätsklinik für Unfallchirurgie, Otto-von-Guericke-Universität Magdeburg, Magdeburg

2 Notfall- und Akutmedizin (CVK, CCM), Charité - Universitätsmedizin Berlin, Berlin

3 Australian Institute of Tropical Health and Medicine, Cairns, James Cook University, Australia

4 Medizinische Fakultät, Institut für Sozialmedizin und Gesundheitssystemforschung, Otto-von-GuerickeUniversität Magdeburg, Magdeburg

5 Fachbereich Gesundheitswesen, Hochschule Niederrhein, Krefeld

6 Wissenschaftliches Institut der AOK (WIdO), Qualitätsund Versorgungsforschung, Berlin

7 Zentralinstitut für die kassenärztliche Versorgung in Deutschland, Berlin

8 Medizinische Fakultät, Universitätsklinik für Anästhesiologie und Intensivtherapie, Otto-von-Guericke-Universität Magdeburg, Magdeburg

Schlüsselwörter

Notaufnahme, Notfallambulanz, Rettungsstelle, Routinedaten, Versorgungsforschung, Kodierte Diagnosen

Key words

Emergency department, Routine data, Administrative data, Health services research, Coded diagnoses

Bibliografie

DOI https://doi.org/10.1055/a-0996-8371

Online-Publikation: 9.10.2019

Gesundheitswesen 2020; 82 (Suppl. 1): S72-S82

(c) Georg Thieme Verlag KG Stuttgart · New York

ISSN 0949-7013
Korrespondenzadresse

Felix Greiner

Universitätsklinik für Unfallchirurgie

Otto-von-Guericke-Universität Magdeburg

Leipziger Straße 44

39120 Magdeburg

felix.greiner@med.ovgu.de

\section{ZUSAMMENFASSUNG}

Hintergrund Nicht nur im Kontext der Neuordnung der Notfallversorgung in Deutschland besteht derzeit ein hoher Bedarf an Daten aus Notaufnahmen. Für die Versorgungsforschung bieten sich Daten an, welche auf gesetzlicher Grundlage generiert werden. Unterschiedliche Kostenträger und Abrechnungsmodi stellen eigene Anforderungen an die Dokumentation dieser Routinedaten.

Methodische Herausforderungen Aufgrund der sektoralen Trennung gibt es keinen Datensatz oder Datenhalter, der Auskunft über alle Notaufnahmebehandlungen geben kann. Aus administrativer Sicht gilt die gesamte Notaufnahmebehandlung als ambulant oder stationär, tatsächlich wird die Entscheidung darüber erst während der Versorgung getroffen. Für die stationäre Versorgung existiert ein administratives Notfallkennzeichen, allerdings kein direktes Merkmal für Notaufnahmebehandlungen. Bei Abrechnung ambulanter Fälle über die kassenärztlichen Vereinigungen ist mindestens eine Diagnose (ICD-10-Kode) zu erfassen, versehen mit einem Kennzeichen zur Diagnosesicherheit. Es können mehrere ICD-10-Kodes ohne Hierarchie angegeben werden. Bei stationär behandelten Patienten ist eine Aufnahmediagnose und nach Behandlungsende die Hauptdiagnose und ggf. Nebendiagose(n) an die zuständige Krankenkasse zu übermitteln. Die gesetzliche Unfallversicherung hat eigene Dokumentationsanforderungen. 
Lösungsansätze Je nach Forschungsfrage und Studiendesign sind unterschiedliche Vorgehensweisen erforderlich. Stammen die Daten unmittelbar aus Notaufnahmen bzw. Kliniken ist eine Information über den Kostenträger und den Abrechnungsmodus hilfreich. Bei Nutzung von Krankenkassendaten muss die Identifikation von stationär behandelten Patienten in einer Notaufnahme aktuell indirekt erfolgen. Dazu können unter anderem die Parameter Aufnahmegrund und definierte „eindeutige“ Notfall-Diagnosen herangezogen werden. Die fallpauschalenbezogene Krankenhausstatistik hat eigene Limitationen, enthält dafür aber die stationären Fälle aller Kostenträger.

Diskussion Die divergierenden Anforderungen an die administrative Dokumentation verursachen einen hohen Aufwand in den Kliniken. Perspektivisch ist eine Vereinheitlichung der Leistungserfassung und Dokumentation von Notfallbehandlungen aller Kostenarten auch zur Generierung von validen, vergleichbaren und repräsentativen Daten für die Versorgungsforschung erstrebenswert. Die Einführung eines eigenen Fachabteilungsschlüssels würde zur Identifikation von Notaufnahmebehandlungen beitragen.

\section{ABSTRACT}

Background Currently, there is a big need for data on emergency department (ED) utilization in Germany. One reason is the ongoing reorganisation of emergency care. Possible sources are routine data that are being collected based on legal regulations. Different payers and compensation systems have their own requirements for data collection.

Methodological challenges Due to the sectoral separation of health care services, there is no dataset or data holder to provide information on all ED treatments in Germany. From an administrative point of view, emergency care in Germany is considered ambulatory outpatient or inpatient care from the time point of admission to the ED. In contrast, clinical decision about inpatient admission can sometimes only be made towards the end of emergency care. EDs themselves cannot be identified in claims data; only the medical discipline (e. g. surgery) is classified. In the case of outpatient treatment, reimbursed by the Association of Statutory Health Insurance Physicians, at least one coded diagnosis (ICD) has to be recorded, accompanied by an additional code for the likelihood of this diagnosis. In case of multiple ICDs, a primary diagnosis cannot be specified. In the case of in-hospital treatment, an admission diagnosis must be recorded. After completion of hospital treatment, the main diagnosis and possibly secondary diagnoses are transferred to the respective health insurance fund. The statutory occupational accident insurance has its own requirements. Solutions Depending on the research question and study design, different approaches are required. If data are queried directly in emergency departments or hospitals, additional information on the designated data holder and billing mode is crucial. When using health insurance data from inpatient care, the identification of emergency departments can be estimated on the basis of the reason for hospital admission and defined "unique" emergency ICDs. The case-related hospital statistics has its own limitations, but includes inpatients of all payers.

Discussion Differing requirements for the administrative documentation cause a high workload in emergency departments. A standardised data collection system for all payers for inpatient and outpatient emergency care is recommended. This would contribute to the creation of valid and comparable datasets. The introduction of a particular identifier for EDs in claims data would enhance health services research.

\section{Hintergrund}

Das Versorgungsgeschehen in Notaufnahmen deutscher Krankenhäuser befindet sich aktuell in einem tiefgreifenden Wandel. Patienten mit akut-, aber nicht notfallmedizinischem Versorgungsbedarf suchen aus vielschichtigen Gründen vermehrt die Notaufnahmen der Krankenhäuser auf [1, 2]. Dort verursachen sie bei steigenden Fallzahlen eine Änderung des Patientenspektrums, wobei auch bei stationär behandelten Patienten eine absolute Zunahme zu beobachten ist [3-6]. Das sogenannte Crowding mit konsekutiver Zunahme der Wartezeiten wird als zunehmendes Problem in den Notaufnahmen wahrgenommen [7-9]. Anbieterseitig werden ehemals fachspezifische Notaufnahmen zu zentralen interdisziplinären Notaufnahmen zusammengeführt $[6,10]$. Für Krankenhäuser, die an der strukturierten Notfallversorgung gemäß Krankenhausstrukturgesetz (KHSG) teilnehmen, wurde die Vorhaltung einer Zentralen Notaufnahme durch Beschluss des Gemeinsamen Bundesausschusses (G-BA) zwischenzeitlich als Standard definiert [11, 12]. Im Kontext der Diskussion um mögliche Fehlanreize der Inanspruchnahme empfiehlt auch der Sachverständigenrat zur Begutachtung der Entwicklung im Gesundheitswesen (SVR Gesundheit) in seinem
Gutachten 2018 eine sektorenübergreifende Neugestaltung der Notfallversorgung [13].

Vor diesem Hintergrund besteht ein hoher Bedarf an Daten zum Versorgungsgeschehen in Notaufnahmen. Eine mögliche Datenquelle für die Versorgungsforschung stellen Routinedaten dar, welche auf gesetzlicher Grundlage im Rahmen der Abrechnung zwischen Leistungserbringern und Kostenträgern oder zur Weiterentwicklung des Fallpauschalen-Vergütungssystems generiert werden $[14,15]$. Unterschiedliche Kostenträger und Abrechnungsmodi stellen jeweils eigene Anforderungen an die Dokumentation von Notaufnahmebehandlungen [16]. Dies gilt insbesondere für die Diagnosekodierung auf Basis der deutschen Version der International Statistical Classification of Diseases and Related Health Problems, 10. Revision, German Modification (ICD-10-GM).

Ziel dieses Artikels ist die Beschreibung ausgewählter Routinedaten, welche von Notaufnahmen im Rahmen der Patientenversorgung obligatorisch zu erfassen sind. Die Unterschiede werden systematisch herausgearbeitet und im Hinblick auf die Nutzung für diagnosebezogene Analysen als auch die Identifizierbarkeit von Notaufnahmepatienten in den verschiedenen Datenquellen bewertet. 


\section{Methodische Herausforderungen}

\section{Datenerhebung in Notaufnahmen}

\section{Schnittstelle Notaufnahme}

Notaufnahmen sind in Krankenhäusern die primäre Anlaufstelle für Notfallpatienten aller Dringlichkeitsstufen und Schweregrade. Diese kommen mit einer Einweisung durch einen niedergelassenen Arzt, ungeplant als „Selbsteinweiser“ oder werden durch den Rettungsdienst eingeliefert. Letztgenannte Möglichkeit beinhaltet auch Fälle mit prähospitaler Notarztversorgung oder dringliche Verlegungen aus anderen Krankenhäusern. Aus klinischer Sicht beginnt die Versorgung eines Patienten in der Notaufnahme mit einem Symptom oder einem Beschwerdekomplex. Erst nach erfolgter Anamnese, körperlicher Untersuchung und weiterer Diagnostik kann das weitere Vorgehen, wie die ärztliche Entscheidung über eine ambulante Behandlung oder stationäre Aufnahme, geplant werden [5, 6, 16, 17]. Das Vorliegen einer Einweisungsdiagnose eignet sich nur bedingt zur Prozessteuerung [18]. Ein Fall kann somit erst retrospektiv als ambulant oder stationär klassifiziert werden ( $>$ Abb. 1). In diesem Artikel wird nachfolgend nur der Begriff Notaufnahme verwendet; weitere gebräuchliche Bezeichnungen sind Notfallambulanz oder Rettungsstelle. Trotz ihrer Semantik erlauben die Begriffe keinen Schluss über eine stationäre Aufnahme oder ambulante Behandlung der dort versorgten Patienten.

Unterschiedliche Perspektiven auf ambulante und stationäre Versorgung

Für die Abgrenzung zwischen ambulanten und stationären Fällen gibt es unterschiedliche Definitionen ( $\triangleright$ Abb. 1). Bei ambulanter Behandlung geht man gemeinhin davon aus, dass der Patient die Notaufnahme direkt wieder verlässt, während die stationäre Behandlung mit mindestens einer Übernachtung im Krankenhaus assoziiert ist. Sektoral wird im deutschen Gesundheitswesen zwi- schen ambulanter (kassenärztlicher) und stationärer Versorgung (Krankenhausbehandlung) unterschieden. In Notaufnahmen sind die Leistungen am Übergang von ambulanter zu stationärer Versorgung fließend [19]. Zu den stationären Entgeltarten gehören gemäß Sozialgesetzbuch (SGB) V auch bestimmte Fälle ohne eine Übernachtung des Patienten im Krankenhaus [20,21]. Dazu zählen in Notaufnahmen Fälle mit vorstationärer Behandlung nach $\S$ 115a SGB V ohne anschließende stationäre Aufnahme („Abklärungsuntersuchung zur Vermeidung von Krankenhausbehandlung“) und die sogenannten pseudostationären oder Stundenfälle (Aufnahme- und Entlassungstag identisch) mit Vergütung nach stationären Fallpauschalen. Bei diesen quasi-ambulanten Fällen wird davon ausgegangen, dass die Ressourcen eines Krankenhauses zur Versorgung notwendig sind [20]. Als vorstationär kann bspw. die Behandlung eines Patienten abgerechnet werden, der die Notaufnahme mit einem Einweisungsschein von seinem Hausarzt aufsucht. Mögliches Szenario wäre der Verdacht auf eine Appendizitis, welcher sich nicht bestätigt. Beispiele für einen vollstationären Stundenfall wäre die Überwachung eines Brustschmerzpatienten über mehrere Stunden auf einer angeschlossenen Überwachungseinheit [22].

\section{Kostenträger und Abrechnungsmodi in Notaufnahmen}

Für Datenerhebung und -übermittlung im Rahmen der Notaufnahmeversorgung gibt es keinen eigenen Rechtsrahmen. Stattdessen kommt jeweils die gesetzliche Grundlage zur Anwendung, über welche die Abrechnung des jeweiligen Falles erfolgt ( $\triangleright$ Tab. 1). In Notaufnahmen werden die meisten Patienten im Rahmen der gesetzlichen Krankenversicherung (GKV) behandelt. Weitere relevante Kostenträger sind die gesetzliche Unfallversicherung (GUV), sofern ein Arbeits- bzw. Wegeunfall die Behandlung verursacht hat, und für Privatversicherte die private Krankenversicherung (PKV).

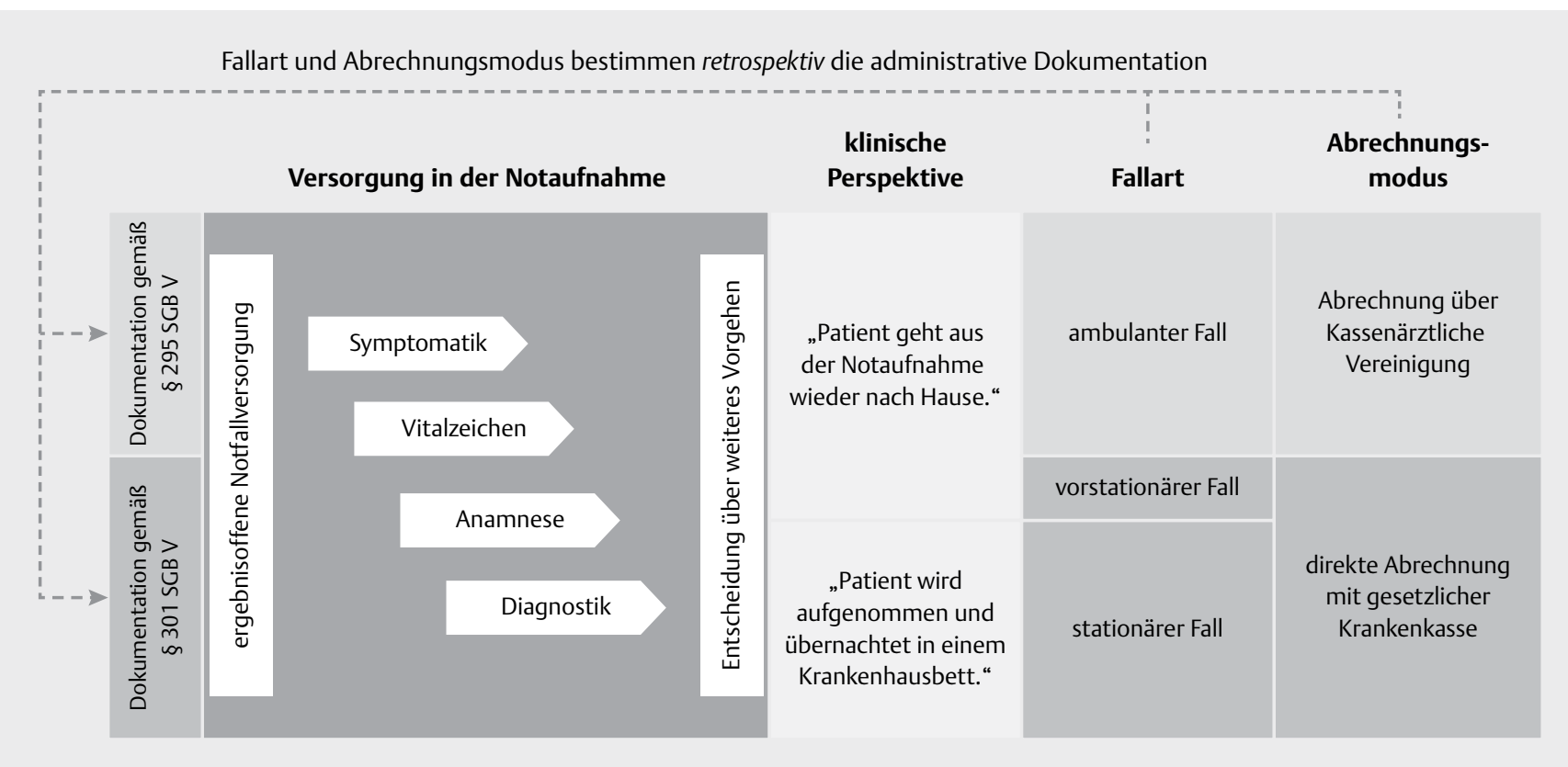

Abb. 1 Unterschiedliche Perspektiven auf ambulante und stationäre Fälle in der klinischen Notfallversorgung sowie Auswirkungen auf die administrative Dokumentation (nur gesetzliche Krankenversicherung). 


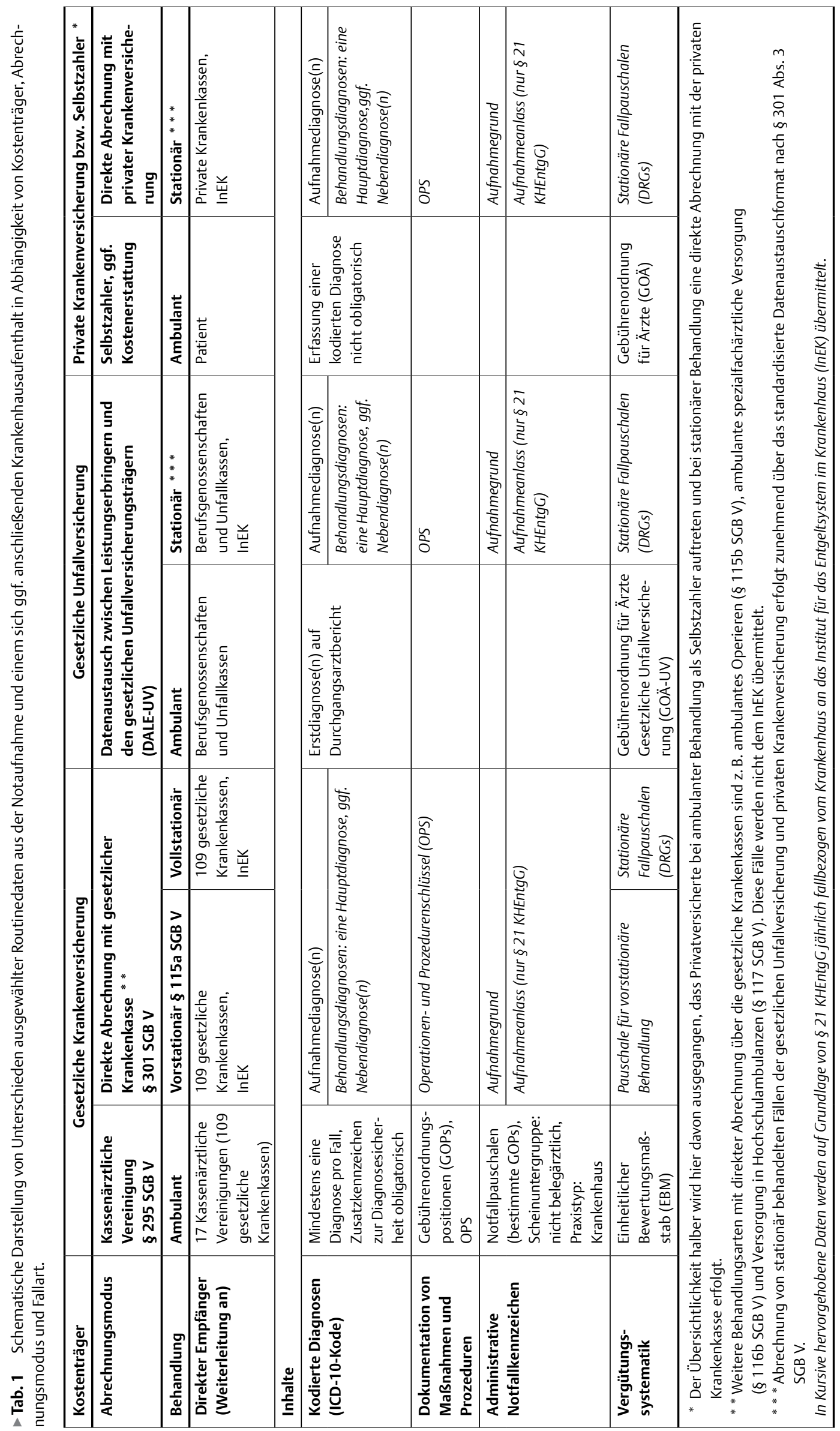


administrative Routinedaten aus der Notaufnahme und einem sich ggf. anschließenden Krankenhausaufenthalt (Erfassung, Verarbeitung und Weiterleitung kann in unterschiedlichen Systemen erfolgen)

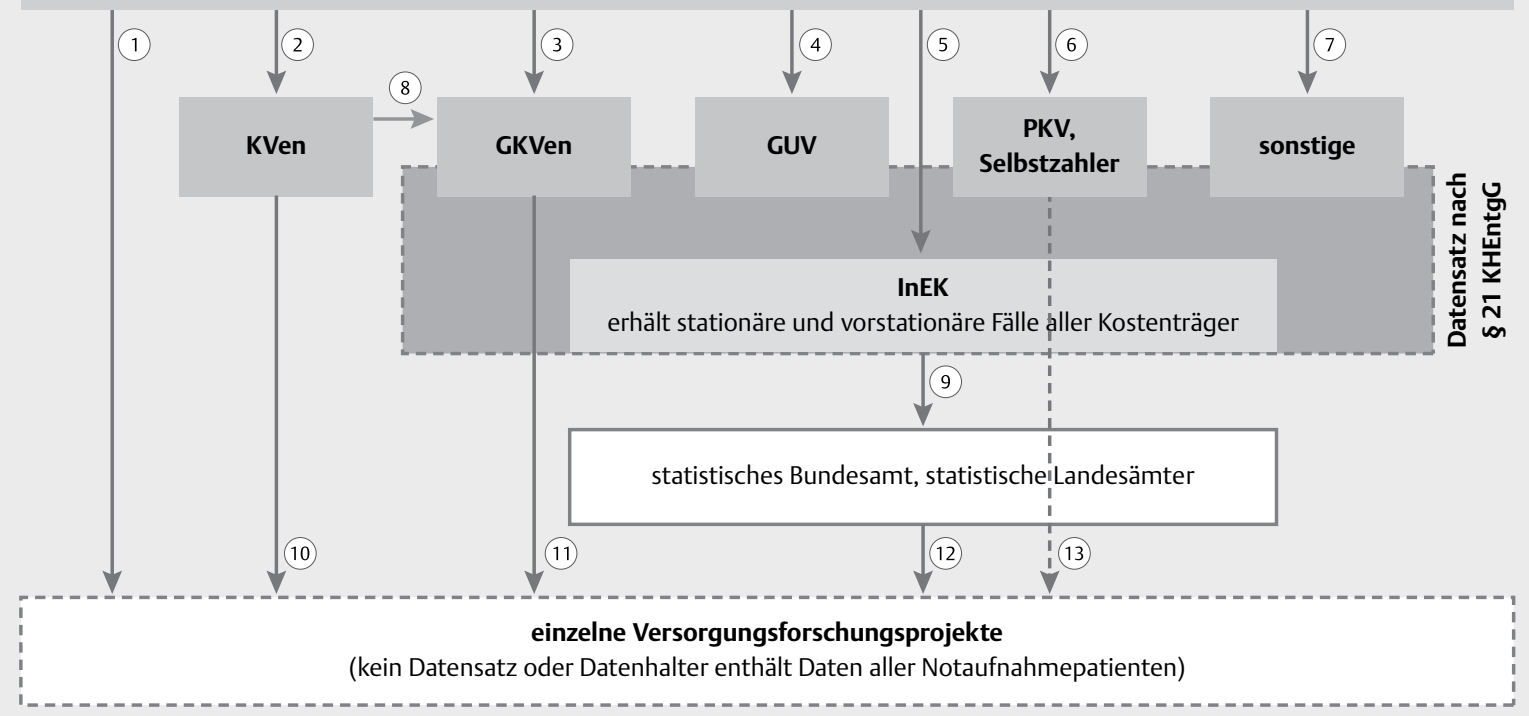

Ausgewählte Besonderheiten zu Datensätzen mit Bezug zu Notaufnahmebehandlungen.

(1) Direkte Abfrage von Daten aller Notaufnahmepatienten ist möglich, vorausgesetzt dass für alle Fälle auch elektronische Daten vorliegen.

(2) Routinedaten von ambulant behandelten Patienten werden quartalsweise an die zuständige kassenärztliche Vereinigung übermittelt.

(3) Routinedaten von stationären und vorstationären Fällen werden direkt an die zuständige gesetzliche Krankenkasse übermittelt (Datenaustausch gemäß § 301 SGB V).

(4) Die primäre Dokumentation von Fällen im Rahmen der gesetzlichen Unfallversicherung erfolgt über den Durchgangsarztbericht (Formular F1000), die Übermittlung über DALE-UV folgt bei stationären Fällen der DTA-Vereinbarung gemäß § 301 SGB V.

(5) Der nach $\S 21 \mathrm{KHEntgG} \mathrm{erhobene} \mathrm{Datensatz} \mathrm{beinhaltet} \mathrm{fast} \mathrm{alle} \mathrm{stationären} \mathrm{und} \mathrm{vorstationären} \mathrm{Fälle} \mathrm{und} \mathrm{wird} \mathrm{jährlich} \mathrm{an} \mathrm{das}$ InEK übermittelt. Dafür wird er in einem standardisierten Exportformat bereitgestellt (mehrere csv-Dateien).

(6) Die Abrechnung kann direkt mit der PKV oder mit dem Patienten erfolgen. Bei Verzicht auf Kostenerstattung durch diesen erfolgt auch keine indirekte Datenübermittlung an die PKV. Für direkte Abrechnung stationärer Fälle gilt die DTA-Vereinbarung gemäß § 301 SGB V.

(7) Hierbei handelt sich z. B. um freie Heilfürsorge der Polizei, unentgeltliche truppenärztliche Versorgung und direkte Abrechnung mit Sozialbehörden.

(8) Versichertenbezogene ambulante Versorgungsdaten werden von kassenärztlichen Vereinigungen an die zuständige gesetzliche Krankenkasse weitergeleitet.

(9) Nach Plausibilitätsprüfung leitet das InEK jährlich ausgewählte fallbezogene Daten gemäß § 21 Abs. 3 KHEntgG an das Statistische Bundesamt weiter.

(10) Daten können prinzipiell anonymisiert oder pseudonymisiert personenbezogen bereitgestellt werden.

(11) Daten können prinzipiell anonymisiert oder pseudonymisiert personenbezogen bereitgestellt werden.

(12) Daten stehen für vollstationäre Fälle prinzipiell anonymisiert fallbezogen zur Verfügung, ansonsten nur aggregiert.

(13) Daten stehen ggf. bei den privaten Krankenkassen oder dem Wissenschaftlichen Institut der PKV (WIP) zur Verfügung.

Die Datennutzung für Versorgungsforschungsprojekte erfordert die entsprechende Bereitschaft der Datenhalter und Einhaltun sämtlicher Datenschutzbestimmungen. Auf Details zum Datenschutz kann an dieser Stelle nicht eingegangen werden.

DALE-UV Datenaustausch zwischen Leistungserbringern und den gesetzlichen Unfallversicherungsträgern,

DTA Datenaustausch,

GKV Gesetzliche Krankenversicherung,

GUV Gesetzliche Unfallversicherung,

InEK Institut für das Entgeltsystem im Krankenhaus,

KHEntgG Krankenhausentgeltgesetz,

KV Kassenärztliche Vereinigung,

PKV Private Krankenversicherung,

SGB Sozialgesetzbuch

Abb. 2 Schema möglicher Datenflüsse und Datenquellen von Routinedaten aus der klinischen Notfallversorgung für die Versorgungsforschung. 
Abrechnung und Datenerhebung ambulanter Notfallbehandlungen (§ 295 SGB V)

Ca. $40 \%$ aller Notaufnahmebehandlungen werden als ambulante Fälle über die Kassenärztlichen Vereinigungen (KVen) abgerechnet $[19,23]$. Die Datenerfassung und -übermittlung folgt hier den Bestimmungen der vertragsärztlichen Versorgung gemäß § 295 SGB V. Für die Datenverarbeitung gilt bei Notfällen ergänzend § 295a Abs. 3 SGB V.

Die Daten werden quartalsweise an die KVen übermittelt ( $\triangleright \mathbf{A b b}$. 2). Für jeden Fall ist mindestens eine nach ICD-10-GM kodierte Diagnose (nachfolgend als ICD-10-Kode bezeichnet) durch den Leistungserbringer zu übermitteln („Abrechnungsdiagnose(n)“). Dabei ist jede Diagnose mit einem von vier Zusatzkennzeichen zur Diagnosesicherheit zu ergänzen (V: Verdacht auf, G: gesichert, A: ausgeschlossen, Z: Zustand nach) [24]. Diese wurden eingeführt, um Leistungen vor Stellen einer Diagnose, bspw. zum Erkrankungsausschluss, zu begründen [25]. Das Zusatzkennzeichen „A“ kehrt die Bedeutung einer Diagnose quasi um, so wird aus dem akuten Myokardinfarkt (ICD-Dreisteller I21) der Ausschluss eines akuten Myokardinfarktes. Ähnliches gilt bei dem ICD10-Kode I21 für das Zusatzkennzeichen „V“ (Verdacht auf akuten Myokardinfarkt). Bei Erfassung mehrerer Diagnosen werden diese gleichwertig übermittelt. Die Kennzeichnung einer „, führenden“ Diagnose ist nicht möglich; dementsprechend lassen sich Patientenkollektive nicht nach ihrer „führenden“ Diagnose gruppieren. In der sonstigen vertragsärztlichen Versorgung lassen sich verschiedene Arztkontakte mit der gleichen Praxis während eines Quartals aufgrund pauschalierter Abrechnungen meist nicht unterscheiden [14]. Im Gegensatz dazu können Notaufnahmekontakte eines Patienten, wie auch Kontakte mit dem kassenärztlichen Notdienst, innerhalb eines Quartals tagesgenau ermittelt werden.

Im Kontext der KV-Abrechnung sind weder Art noch Anzahl der Diagnosen pro Behandlungsfall direkt erlösrelevant, sondern beeinflussen gemäß § 87a SGB V die morbiditätsbedingte Gesamtvergütung im vertragsärztlichen Bereich. Auf Fallebene dienen einzelne Diagnosen zur Darlegung einer medizinischen Indikation für durchgeführte Leistungen. Beispielsweise begründet der ICD-10-Kode I61 „A“ (Ausschluss einer intrazerebralen Blutung) eine durchgeführte craniale Computertomografie (CCT).

Im Rahmen der KV-Abrechnung ist gemäß § 295 SGB V die Anwendung des Operationen- und Prozedurenschlüssels (OPS) vorgesehen. Allerdings sind diese Angaben für die Abrechnung kaum von Bedeutung. Zudem sind zahlreiche ambulant zu erbringende Maßnahmen hier nicht gelistet. Eine größere Relevanz weisen Gebührenordnungspositionen (GOP) nach Einheitlichem Bewertungsmaßstab (EBM) auf, welche Behandlungen für die Versorgung im Notfall dokumentieren. Je nach Tag und Zeit der Inanspruchnahme kommen bspw. die Notfallpauschalen mit GOP 01210 oder 01212 zum Einsatz [26]. Die GOP 01205 und 01207 wurden im April 2017 eingeführt und sind als „Notfallpauschale (Abklärung, Koordination)“ abzurechnen, „wenn die Erkrankung des Patienten aufgrund ihrer Beschaffenheit keiner sofortigen Maßnahme bedarf und die nachfolgende Versorgung durch einen Vertragsarzt außerhalb der Notfallversorgung möglich und/oder aufgrund der Umstände vertretbar ist“ [27]. Die Daten werden durch die KVen auf sachlichrechnerische Richtigkeit geprüft. Seit 2004 werden die ambulanten Behandlungsdaten versichertenbezogen an die zuständigen Krankenkassen weitergeleitet [14].
Abrechnung und Datenerhebung stationärer Notfallbehandlungen (§ 301 SGB V)

Stationäre Behandlungen fallen in den Geltungsbereich des $\S 301$ SGB V. In diesem Fall sind die gesetzlichen Krankenkassen direkt zahlungspflichtig ( $\triangleright$ Tab. 1). Die Übermittlung von Aufnahmesatz und Entlassungsanzeige soll innerhalb von drei Arbeitstagen an die zuständige gesetzliche Krankenkasse erfolgen, der Rechnungssatz in der Regel einmal pro Kalenderwoche [21].

Für jeden GKV-Fall ist mindestens eine Aufnahmediagnose Bestandteil der Aufnahmeanzeige; theoretisch sind bis zu 20 Aufnahmediagnosen möglich. Die Behandlungsdiagnosen werden nach Ende des stationären Aufenthaltes mit der Entlassungsanzeige an die zuständige gesetzliche Krankenkasse übermittelt ( $\triangleright \mathbf{A b b} . \mathbf{2}$ ). Hierbei ist für den gesamten Krankenhausaufenthalt eine Hauptdiagnose zu definieren, die hauptsächlich für die Veranlassung des stationären Aufenthaltes verantwortlich ist [28]. Bei aufeinanderfolgenden stationären Aufenthalten sachlicher Ähnlichkeit innerhalb definierter Zeitfristen kann eine Fallzusammenführung notwendig werden, bspw. wenn der zweite Aufenthalt aufgrund von Komplikationen im Anschluss an den ersten Aufenthalt erfolgt [29]. Dann werden die Diagnosen beider Aufenthalte zusammengefasst und es wird nur ein Fall mit einer Hauptdiagnose abgerechnet.

Die ICD-10-Kodierung der Diagnosen und Verschlüsselung von Maßnahmen über OPS hat im stationären Bereich unter Beachtung der Deutschen Kodierrichtlinien zu erfolgen [28]. Neben der Überoder Unterschreitung der Verweildauergrenzen beeinflussen sowohl die konkrete Hauptdiagnose als auch die Art und die Anzahl der Nebendiagnosen sowie die nach OPS kodierten Leistungen die Höhe der Fallpauschale. Daraus ergibt sich eine direkte Erlösrelevanz. Gemäß § 275 SGB V ist eine Überprüfung durch den Medizinischen Dienst der Krankenversicherung möglich.

\section{Vorstationäre Behandlung und ambulantes Operieren}

Auch bestimmte ambulante Notfallleistungen werden direkt von den Krankenkassen vergütet. Dazu zählen vorstationäre Behandlungen ohne stationäre Aufnahme (§ 115a SGB V). Diese Fälle sind in den Routinedaten der gesetzlichen Krankenkassen verfügbar ( Tab. 1). Ambulantes Operieren im Krankenhaus nach § 115b SGB $\checkmark$ wird direkt über die Krankenkassen und nur bei belegärztlicher Durchführung über die Kassenärztlichen Vereinigungen abgerechnet [30].

Gesetzliche Unfallversicherung, private Krankenversicherung und Selbstzahler

Arbeits- und Wegeunfälle fallen unter die Vorschriften für die GUV, die sich im SGB VII finden. Bei Behandlungen im Rahmen der GUV erfolgt die Erhebung der so genannten „Erstdiagnose(n)“ mit dem standardisierten Durchgangsarztbericht (Formular F1000) der GUV für ambulant wie auch stationär behandelte Patienten ( Tab. 1). Wie bei der KV-Abrechnung werden mehrere Diagnosen gleichwertig übermittelt; die Festlegung einer „führenden“ Erstdiagnose ist nicht vorgesehen. Ambulante Behandlungen werden über die Gebührenordnung für Ärzte Gesetzliche Unfallversicherung (GOÄUV) abgerechnet, der Datenaustausch erfolgt elektronisch über DALE-UV (Datenaustausch zwischen Leistungserbringern und den gesetzlichen Unfallversicherungsträgern). Für Abrechnungen mit der PKV oder bei Selbstzahlern ist nach ambulanter Behandlung 
die Erfassung eines ICD-10-Kodes nicht obligatorisch. Hier gilt die Gebührenordnung für Ärzte (GOÄ).

Die Abrechnung und somit auch Dokumentation von stationär behandelten Fällen der GUV und PKV folgt zunehmend dem standardisierten Datenaustauschformat nach $\S 301$ Abs. 3 SGB V $[15,31]$.

\section{Datenerhebung gemäß Krankenhausentgeltgesetz}

Eine weitere Verpflichtung zur Datenerhebung im Kontext der stationären Versorgung ergibt sich aus den Anforderungen zur Weiterentwicklung des Fallpauschalen-Vergütungssystems gemäß § 21 Krankenhausentgeltgesetz (KHEntgG). Die fallbezogenen Datenelemente nach § 21 KHEntgG gleichen zu einem großen Teil den Inhalten der nach $\S 301$ SGB V erhobenen Daten [21, 32]. Sie sind jährlich von den Krankenhäusern an das Institut für das Entgeltsystem im Krankenhaus (InEK) zu übermitteln ( $\triangleright$ Abb. 2). Bis auf wenige Ausnahmen (z. B. GUV-Fälle aus Kliniken der Berufsgenossenschaften und ausländische Selbstzahler) sind hier alle stationären Krankenhausfälle enthalten, also auch solche mit PKV oder der GUV als Kostenträger [15], ebenso vorstationäre Fälle oder stationäre Stundenfälle. Im Gegensatz zu den Daten gemäß § 301 SGB V sind nur die Behandlungsdiagnosen Bestandteil des Datensatzes gemäß $\S 21 \mathrm{KHEntgG}$, nicht aber die Aufnahmediagnosen ( $>$ Tab. 1). Ein weiterer Unterschied ist, dass Prozeduren (OPS) mit Zeitstempel übermittelt werden, während im § 301-Verfahren nur eine tagesgenaue Angabe vorgesehen ist [21, 32]. Die Daten des InEK werden an das Statistische Bundesamt weitergeleitet und dort als Basis für die fallpauschalenbezogene Krankenhausstatistik genutzt, welche allerdings nur fallbezogene Daten der vollstationären Behandlungen enthält [33].

\section{Limitationen diagnosebezogener Analysen}

Zusammengefasst ergeben sich insbesondere bei diagnosebezogenen Analysen ambulanter Notaufnahmefälle erhebliche Limitationen. Hier ist bei GKV- und GUV-Fällen weder die Kennzeichnung einer „führenden“ Diagnose, noch bei PKV-Fällen bzw. Selbstzah- lern überhaupt eine Erfassung von ICD-10-Kodes erforderlich ( $>$ Tab. 1). Bei gleicher Behandlung stellt ein nach einem Unfallereignis ambulant versorgter Fall unterschiedliche Anforderungen an die Erfassung von Daten, je nachdem ob es sich um einen Unfall in der Freizeit (GKV: ICD-10-Kodes mit Zusatzkennzeichen) oder während der Arbeitszeit (GUV: ICD-10-Kodes ohne Zusatzkennzeichen) handelt ( $\mathbf{A} \mathbf{\text { bb }}$. $\mathbf{3}$ ). Innerhalb der GKV kann neben dem Versorgungsaufwand auch die Zuweisungsart darüber entscheiden, ob die Behandlung eines Patienten als ambulanter KV-Fall oder vorstationärer Fall zur Abrechnung kommt [10]. Im stationären Sektor müssen im Abrechnungsdatensatz weder die Aufnahme- noch die Behandlungsdiagnose exakt mit dem Behandlungsanlass in der Notaufnahme übereinstimmen. Die Dokumentation einer rein medizinischen „Notaufnahmediagnose“ ist generell nicht vorgesehen. Grundsätzlich wird bei allen diagnosebezogenen Analysen die exante Sicht der klinischen Versorgung nicht berücksichtigt, nämlich dass Notfälle ausgehend von Symptomen und Dringlichkeit behandelt werden.

\section{Administrativer Aufwand für Notaufnahmen}

In den Krankenhäusern wird die Dokumentation und Abrechnung für die unterschiedlichen Kostenträger und Abrechnungsmodi aufgrund gewachsener Strukturen häufig von unterschiedlichen Mitarbeitern, gelegentlich in verschiedenen Abteilungen und sogar in verschiedenen Informationssystemen bearbeitet. Einzelne Notaufnahmen kodieren zwar für alle Patienten eine medizinische „Notaufnahmediagnose “ unabhängig von der antizipierten Abrechnungsart; diese muss dann aber bei Nutzung für die Abrechnung noch administrativ modifiziert werden, z. B. durch Ergänzung der Zusatzkennzeichen (V, G, A, Z) bei Abrechnungen über die KVen. Teilweise erforderliche Wandlungen von Kostenträger und Abrechnungsmodus betreffen jeweils die administrative Dokumentation ab Behandlungsbeginn ( $>$ Abb. 1). Unter den geschilderten Rahmenbedingungen ist selbst für einzelne Krankenhäuser die Erstellung eines integrierten Datensatzes zu allen Notaufnahmepatienten mit extrem hohem Aufwand verbunden.

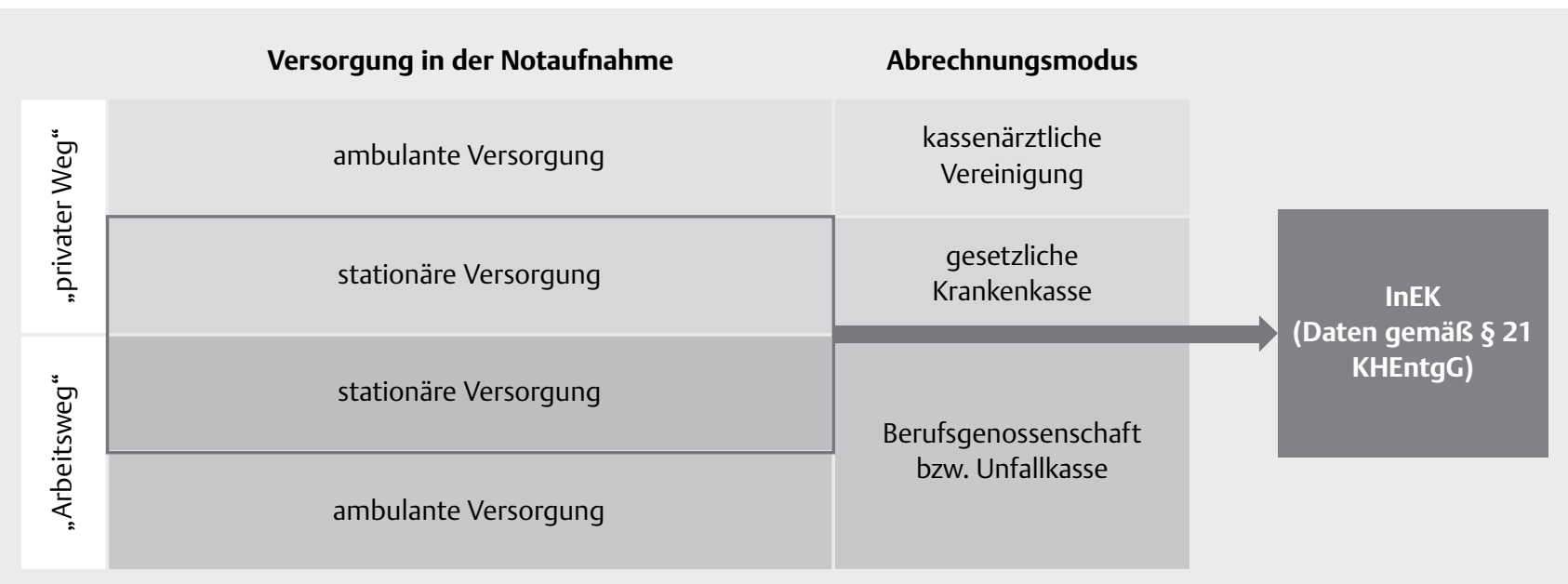

Abb. 3 Unterschiedliche Abrechnungsmodi und Datenhalter bei einem Wegeunfall mit gleichem Verletzungsmuster und identischer Versorgung in der Notaufnahme (am Beispiel von sozialversicherungspflichtig Beschäftigten). 


\section{Identifikation von Notaufnahmen als Ort der Behandlung}

Wie bisher beschrieben divergiert in den Notaufnahmen und Krankenhäusern die fallbezogene Datenerhebung in Abhängigkeit von Kostenträger und Abrechnungsmodus. Trotzdem liegen hier Behandlungsdaten für alle Notaufnahmepatienten vor. Im Rahmen der Abrechnung werden die Daten der Patientenkollektive getrennt und an eine der 109 gesetzlichen Krankenkassen (Stand 2019), eine der 17 KVen, einen Kostenträger der GUV oder PKV bzw. jährlich an das InEK weitergeleitet. Es gibt keinen Datensatz oder Datenhalter, der einen Überblick über die Behandlungsdaten aller Notaufnahmepatienten in ganz Deutschland geben kann ( $\mathbf{A} \mathbf{b} \mathbf{b} . \mathbf{2}$ ). Bei Nutzung einer der genannten Routinedatenquellen für die Versorgungsforschung stellt sich die Frage der Identifizierbarkeit von Notaufnahmen als Ort der Inanspruchnahme.

Eine Notaufnahme kann über eine eigene Betriebsstättennummer (BSNR) gemäß der vertragsärztlichen Vorgaben verfügen [34]. In diesem Fall können in den vertragsärztlichen Abrechnungsdaten ambulante Behandlungen in einer Notaufnahme über diese Nummer direkt identifiziert werden. Die gemeinsame Nutzung einer BSNR mit anderen Struktureinheiten in der Klinik, wie z. B. einer fachspezifischen Hochschulambulanz, ist jedoch möglich, sodass dieses Merkmal nicht eindeutig ist. Eine Filtermöglichkeit für Notaufnahmen ist in der BSNR-Systematik nicht gegeben. Über die oben genannten GOP lassen sich Notaufnahmebesuche nicht eindeutig identifizieren, da diese Notfallpauschalen auch im Rahmen des kassenärztlichen Notdienstes zur Anwendung kommen.

In Daten gemäß § 301 SGB V und § 21 KHEntgG existiert kein direktes Merkmal für Notaufnahmebehandlungen wie bspw. ein eigener Fachabteilungsschlüssel [6]. Allerdings lässt sich im Aufnahmegrund mit dem Schlüssel „xx07“ ein Notfall von einem Normalfall „xx01“ abgrenzen [21]. Mit dem Aufnahmeanlass „N“ Notfall existiert in den Daten gemäß § $21 \mathrm{KHEntgG}$ ein weiteres Kennzeichen [32]. Die Vergabe dieser Kennzeichen hat keine direkte Erlösrelevanz und ist weder trennscharf definiert noch aus medizinischer Sicht überlappungsfrei mit anderen Kategorien, wie bspw. mit dem Aufnahmegrund „xx02“ Arbeitsunfall. Auch ist es möglich, dass Notfallpatienten durch einen Vertragsarzt eingewiesen werden. Somit lässt sich konstatieren, dass die administrative und medizinische Sicht auf einen Notfall unterschiedlich sein kann [35]. Aufgrund diverser Datenhalter und unterschiedlicher Identifizierbarkeit in Sekundärdaten gestalten sich Prävalenzschätzungen von Behandlungen in Notaufnahmen demnach als schwierig.

\section{Lösungsansätze}

Sowohl Daten der KVen und gesetzlicher Krankenkassen als auch Daten des InEK lassen sich für wissenschaftliche Zwecke im Kontext der Notfallversorgung nutzen [4, 26, 35, 36]. Im Rahmen der Versorgungsforschung mit Routinedaten aus Notaufnahmen erfordern Studienplanung und Analyse jedoch eine genaue Kenntnis der Inhalte, Unterschiede und Limitationen der einzelnen Datenquellen, insbesondere für die Auswahl geeigneter Routinedatenquellen. Stationär versorgte Notfallpatienten aller Kostenträger können deutschlandweit mit Daten gemäß § 21 KHEntgG untersucht werden ( $\triangleright$ Abb. 2), bei Abfrage über das Statistische Bundesamt allerdings nur ohne vorstationäre Fälle [33].

\section{Eindeutige Operationalisierung der Fallart}

Bei Nutzung der Begriffe ambulant und stationär ist eine eindeutige und trennscharfe Definition und Operationalisierung erforderlich. Vorstationäre Fälle ohne anschließende vollstationäre Behandlung können je nach Fragestellung einem der beiden Kollektive zugeordnet werden. In Daten gemäß § 301 SGB V sind diese Fälle mit dem Schlüssel „04xx“ im Aufnahmegrund explizit als solche ausgewiesen [21].

\section{Berücksichtigung von Kostenträger und} Abrechnungsmodus

Bei direkter Abfrage von Notaufnahmebehandlungsdaten aus Krankenhäusern für diagnosebasierte Analysen sollte die Zuordnung zu einem Kostenträger und Abrechnungsmodus grundsätzlich mitberücksichtigt werden. Medizinische Diagnosen ohne administrativen Bezug sollten als solche erkennbar sein. Gleiches gilt für die Differenzierung zwischen Aufnahme- sowie Haupt- und Nebendiagnosen. Diese Attribute ermöglichen Rückschlüsse auf die Validität der ICD-10-Kodes und Hinweise auf weitere zu berücksichtigende Informationen wie die Zusatzkennzeichen ( $\mathrm{V}, \mathrm{G}, \mathrm{A}, \mathrm{Z}$ ) bei Abrechnung über die KVen. Als qualifizierendes Diagnosemerkmal liegen sie in einem eigenen Datenfeld vor und müssen explizit abgefragt werden.

\section{Standardisierung klinischer Dokumentation und Nutzung in einem Register}

Bei direkter Datenabfrage aus einem Notaufnahmeinformationssystem können alle Behandlungsfälle unabhängig von Kostenträger und Fallart erfasst werden. In einer Machbarkeitsstudie wird im Projekt AKTIN „Verbesserung der Versorgungsforschung in der Akutmedizin in Deutschland durch den Aufbau eines nationalen Notaufnahmeregisters“ (BMBF-Förderkennzeichen: 01KX1319) der datenschutzkonforme Zugriff auf Notaufnahmedaten über eine dezentrale Register-Infrastruktur erarbeitet [37, 38]. Primär geht es um die elektronische Umsetzung einer standardisierten medizinischen Dokumentation auf Basis des Datensatzes Notaufnahme („Notaufnahmeprotokoll“), der neben klinischen Parametern wie Symptomatik, Stufe der Ersteinschätzung, Vitalzeichen, durchgeführte Diagnostik und klinische Notaufnahmediagnosen auch administrative Informationen wie Zuweisung, Behandlungsdauer und Daten zu dem Verbleib der Patienten enthält [39]. Die Implementierung von Abrechnungs- und Fallart steht noch aus, ist aber für die Bildung von Vergleichskollektiven zu den vorhandenen Sekundärdatenquellen erforderlich.

\section{Identifizierbarkeit von Notaufnahmebehandlungen}

Bei Nutzung von Daten nach § 301 SGB V (der gesetzlichen Krankenkassen) oder Daten nach § $21 \mathrm{KHEntgG} \mathrm{(InEK)} \mathrm{müssen} \mathrm{aufgrund}$ des fehlenden Merkmals für Notaufnahmebehandlungen andere Parameter zur Identifikation stationär behandelter Notaufnahmepatienten herangezogen werden. Über die Parameter Aufnahmegrund „xx07 - Notfall“ bzw. Aufnahmeanlass „N - Notfall“ lassen sich Fälle identifizieren, die administrativ als Notfälle geführt werden. Die Notfalldefinition über den Aufnahmeanlass kann möglicherweise bedeuten, dass der Zugang in das Krankenhaus über die Notaufnahme erfolgte. Allerdings ist aus den oben geschilderten 
Gründen unklar, inwieweit dieses Merkmal bundesweit einheitlich verwendet wird. Zur Identifikation von Notfallbehandlungen wurden weitere Kriterien vorgeschlagen, die auf die Aufnahmeuhrzeit, Notfall-Behandlungsanlässe (Notfall-Diagnosen) und die zeitliche Abfolge von diagnostischen und therapeutischen Maßnahmen Bezug nehmen [35, 40, 41].

Bei Nutzung von KV-Daten oder Abfrage vertragsärztlicher Daten bei den gesetzlichen Krankenkassen besteht ein Identifizierungsansatz über abgerechnete Notfallpauschalen nach EBM (GOP 01210, 01210 ff.) in Kombination mit der BSNR der Notaufnahme. Über die Abklärungspauschalen (GOP 01205 und 01207) und BSNR lassen sich Notaufnahmekontakte ohne dortige Behandlung identifizieren.

\section{Diskussion und Ausblick}

Routinedaten weisen in Bezug auf die zugrundeliegende Grundgesamtheit in der Regel einen geringen Stichprobenfehler und damit eine vergleichsweise hohe Generalisierbarkeit auf. Überdies lassen sich hohe Fallzahlen und lange Beobachtungszeiträume abbilden [42]. Routinedaten aus Notaufnahmen haben die Besonderheit, dass sie zwischen den Vergütungssystemen der Versorgungssektoren divergieren ( $\triangleright$ Tab. 1). Daher sind sie aktuell nur mit hohem Aufwand nutzbar.

Neben Etablierung klinischer Dokumentationsstandards ist nicht nur für Zwecke der Versorgungsforschung auf Basis valider Routinedaten auch eine Vereinheitlichung der administrativen Dokumentation in den Notaufnahmen zu fordern. Die Regelungen des G-BA zu einem gestuften System von Notfallstrukturen in Krankenhäusern sehen vor, dass die Notfallversorgung dort überwiegend in einer Zentralen Notaufnahme erfolgen soll. Selbige ist eine räumlich abgegrenzte, fachübergreifende Einheit mit eigenständiger und fachlich unabhängiger Leitung [12]. Damit wäre die Voraussetzung zur Einführung eines eigenständigen Fachabteilungsschlüssels für Notaufnahmen erfüllt. Dieser würde in Routinedaten die Zuordnung von ICD-10-Kodes und OPS zu einer Notaufnahmebehandlung ermöglichen und gleichzeitig die Identifizierung von Notaufnahmen als Ort der Leistungserbringung sicherstellen $[6,43]$.

Gemäß KHSG ist eine stärkere Verzahnung von stationärer und kassenärztlicher Notfallversorgung vorgesehen, bspw. durch Einrichtung von Portalpraxen an Krankenhäusern. Der SVR Gesundheit empfiehlt darüber hinaus die Einrichtung von interdisziplinär und sektorenübergreifend organisierten sogenannten Integrierten Notfallzentren (INZ). Diese räumlichen und technisch-organisatorischen Einheiten sollen in gemeinsamer Trägerschaft und betrieblicher Verantwortung durch das Krankenhaus und die jeweilige KV betrieben werden. Eine standardisierte digitale Dokumentation soll den sektorenübergreifenden Datenaustausch ermöglichen [13]. Eine Einigung auf einen gemeinsamen Dokumentationsstandard in allen oben genannten Strukturen ist zwingend erforderlich. Über die Einbeziehung von Symptomen bzw. Vorstellungsgründen und der Stufe der Ersteinschätzung (Triage) ließe sich im Gegensatz zu diagnosebasierten Methoden die ex-ante Sicht der klinischen Realität abbilden. Gleichzeitig beeinflussen diese Parameter bei ambulanten Notaufnahmefällen die Kosten [44]. Mit dem CEDIS-Katalog für Vorstellungsgründe liegt eine international gültige
Symptomklassifikation vor, welche bereits im AKTIN-Notaufnahmeregister genutzt wird $[16,45,46]$.

Der einheitliche Dokumentationsstandard für den ambulanten und stationären Sektor sollte so ausgestaltet sein, dass er sich neben Leistungserfassung und Abrechnungsgrundlage auch im Kontext der Qualitätssicherung nutzen lässt $[13,43]$. Eine einheitliche Datenbasis würde die Beantwortung versorgungsepidemiologischer Fragestellungen im Kontext der Notfallbehandlung verbessern und nicht zuletzt den administrativen Aufwand für die beteiligten Leistungserbringer verringern.

\section{FAZIT FÜR DIE PRAXIS}

- Es gibt keinen Datensatz oder Datenhalter, der Auskunft über alle Notaufnahmepatienten in Deutschland geben kann.

- Die Identifizierung von Notaufnahmeaufenthalten muss teilweise indirekt erfolgen. Durch Einführung eines eigenen Fachabteilungsschlüssels wäre eine direkte Zuordnung der dort behandelten Fälle möglich.

- In administrativen Routinedaten ist die gesamte Notaufnahmebehandlung entweder ambulant oder stationär. Das widerspricht der klinischen Realität, wo die Zuordnung zu einer Fallart oft erst nach durchgeführter Diagnostik und Therapie möglich ist.

- Die Bedeutung der Begriffe ambulant und stationär ist in Studienprotokollen genau zu operationalisieren. Je nach Fragestellung können zu ambulant behandelten Patienten in Notaufnahmen nur die KV-Fälle oder auch vorstationäre und ggf. sogar vollstationäre Stundenfälle gezählt werden.

- Bei kodierten Diagnosen aus der Notfallversorgung sollte immer das Vorhandensein von Zusatzkennzeichen ( $\mathrm{V}, \mathrm{G}, \mathrm{A}, \mathrm{Z})$ vermutet werden, da diese integraler Bestandteil des ICD-10-Kodes bei KV-Fällen sind. Sie liegen in einem eigenen Datenfeld vor.

- Die Erfassung von nach ICD-10-GM kodierten Diagnosen wird durch Kostenträger und Abrechnungsmodus beeinflusst.

\section{Danksagung}

Wir bedanken uns bei Steffi Kaufmann und Jannett Katies vom Universitätsklinikum Magdeburg A. ö. R. sowie Dr. Bernadett Erdmann aus der Zentralen Notfallaufnahme am Klinikum Wolfsburg für wertvolle Detailinformationen sowie administrative und ärztliche Expertise zu komplexen Sachverhalten.

Interessenkonflikt

Die Autoren geben an, Förderung im Kontext folgender Projekte erhalten zu haben: F. Greiner: AKTIN (Bundesministerium für Bildung und Forschung, 01KX1319) und INDEED (Innovationsfonds, 01VSF16044). A. Slagman: Forschungsstipendium der Deutschen Forschungsgemeinschaft, Fördermittel des Zentralinstitutes für die 
Kassenärztliche Versorgung, öffentliche Fördermittel des BMBF (EMANET, LIFE-POC) und des Innovationsfonds (INDEED) sowie industrielle Fördermittel (Thermo Fisher Scientific, Novartis, Roche Diagnostics, Radiometer). S. March wird im Rahmen der EVA64-Studie, der Bundesweit einheitlichen Wissenschaftlichen Evaluation von Modellprojekten nach § 64b SGB V, finanziert. Die Förderung der EVA64-Studie erfolgt von einem Konsortium bestehend aus 89 Gesetzlichen Krankenkassen. J. Pollmanns: AKTIN und ENQuIRE (Innovationsfonds, 01VSF17005). P. Dröge: INDEED und OPTINOFA (Innovationsfonds, 01NVF17035). D. Brammen: AKTIN. S. Drösler ist Antragstellerin der Projekte AKTIN und ENQuIRE. F. Walcher ist Hauptantragsteller der Projekte AKTIN und ENQuIRE. J. Heuer und M.-L. Rosenbusch sind Angestellte des Zentralinstituts für die kassenärztliche Versorgung in Deutschland (Zi). Das Zi ist eine Stiftung des bürgerlichen Rechts, die von 17 Kassenärztlichen Vereinigungen (KVen) und der Kassenärztlichen Bundesvereinigung getragen wird. Satzungsgemäß unterstützt die Forschung des Zi die KVen bei der Wahrnehmung ihres gesetzlichen Sicherstellungsauftrages. Bei J. Heuer und M.-L. Rosenbusch besteht kein Interessenskonflikt durch anderweitige Beraterhonorare oder Beschäftigungsverhältnisse. C. Stallmann und C. Günster geben an, dass kein Interessenkonflikt besteht.

\section{Literatur}

[1] Schmiedhofer MH, Searle J, Slagman A et al. Inanspruchnahme zentraler Notaufnahmen: Qualitative Erhebung der Motivation von Patientinnen und Patienten mit nichtdringlichem Behandlungsbedarf. Gesundheitswesen 2017; 79: 835-844. doi:10.1055/s-0042-100729

[2] Somasundaram R, Geissler A, Leidel BA et al. Beweggründe für die Inanspruchnahme von Notaufnahmen - Ergebnisse einer Patientenbefragung. Gesundheitswesen 2018; 80: 621-627. doi:10.1055/s-0042-112459

[3] Pines JM, Hilton JA, Weber E] et al. International perspectives on emergency department crowding. Acad Emerg Med 2011; 18: 1358-1370. doi:10.1111/j.1553-2712.2011.01235.x

[4] Dräther H, Schäfer T. Die ambulante Notfallversorgung in Notfallambulanzen und bei Vertragsärzten im Zeitraum 2009 bis 2014. In: Klauber J, Geraedts M, Friedrich J et al., Hrsg. Krankenhaus-Report 2017: Zukunft gestalten. Stuttgart: Schattauer; 2017: 25-40

[5] Fleischmann T. Schnittstelle Zentrale Notaufnahme. Dtsch Med Wochenschr 2016; 141: 19-23. doi:10.1055/s-0041-108906

[6] Christ M, Dodt C, Geldner G et al. Professionalisierung der klinischen Notfallmedizin - Gegenwart und Zukunft. Anasthesiol Intensivmed Notfallmed Schmerzther 2010; 45: 666-671. doi:10.1055/s-0030-1267533

[7] Bernstein SL, Aronsky D, Duseja R et al. The Effect of Emergency Department Crowding on Clinically Oriented Outcomes. Acad Emerg Med 2009; 16: 1-10. doi:10.1111/j.1553-2712.2008.00295.x

[8] Trzeczak S. Überfüllte Notaufnahme. Notfall + Rettungsmedizin 2013; 16: 103-108. doi:10.1007/s10049-012-1625-2

[9] Searle J, Muller R, Slagman A et al. Überfüllung der Notaufnahmen. Notfall + Rettungsmedizin 2015; 18: 306-315. doi:10.1007| s10049-015-0011-2

[10] Schöpke T, Plappert T. Kennzahlen von Notaufnahmen in Deutschland. Notfall Rettungsmed 2011; 14: 371-378. doi:10.1007/s10049-011$1435-y$

[11] Bundestag. Gesetz zur Reform der Strukturen der Krankenhausversorgung (Krankenhausstrukturgesetz - KHSG). Bundesgesetzblatt 2015; Bundesgesetzblatt Jahrgang 2015; Teil I Nr. 51

[12] Bundesministerium für Gesundheit. Bekanntmachung eines Beschlusses des Gemeinsamen Bundesausschusses über die Erstfassung der Regelungen zu einem gestuften System von Notfallstrukturen in Krankenhäusern gemäß § 136c Absatz 4 des Fünften Buches Sozialgesetzbuch (SGB V). Bundesanzeiger 2018 BAnz AT 18.05.2018 B4
[13] Sachverständigenrat zur Begutachtung der Entwicklung im Gesundheitswesen. Bedarfsgerechte Steuerung der Gesundheitsversorgung. 2018

[14] Grobe TG, Dräther H, Ambulante ärztliche Versorgung. In: Swart E, Ihle P, Gothe $\mathrm{H}$ et al., Hrsg. Routinedaten im Gesundheitswesen: Handbuch Sekundärdatenanalyse: Grundlagen, Methoden und Perspektiven. Bern: Verlag Hans Huber; 2014

[15] Grobe TG, Nimptsch U, Friedrich J, Krankenhausbehandlung. In: Swart E, Ihle P, Gothe $\mathrm{H}$ et al. Hrsg. Routinedaten im Gesundheitswesen: Handbuch Sekundärdatenanalyse: Grundlagen, Methoden und Perspektiven. Bern: Verlag Hans Huber; 2014

[16] Greiner F, Brammen D, Routinedatenbasierte Versorgungsforschung in der klinischen Notfallmedizin - Herausforderungen und Möglichkeiten. In: Klauber J, Geraedts M, Friedrich J et al. Hrsg. KrankenhausReport 2018: Bedarf und Bedarfsgerechtigkeit. Stuttgart: Schattauer; 2018: 259-272

[17] Möckel M, Searle J, Muller R et al. Chief complaints in medical emergencies: do they relate to underlying disease and outcome? The Charite Emergency Medicine Study (CHARITEM). Eur J Emerg Med 2013; 20: 103-108. doi:10.1097/MEJ.0b013e328351e609

[18] Bernhard M, Raatz C, Zahn P et al. Validität von Einweisungsdiagnosen als Prozesssteuerungskriterium. Der Anaesthesist 2013; 62: 617-623. doi:10.1007/s00101-013-2207-5

[19] Haas C, Larbig M, Schöpke T et al. Gutachten zur ambulanten Notfallversorgung im Krankenhaus - Fallkostenkalkulation und Strukturanalyse. Hamburg: MCK und DGINA; 2015

[20] Leber W-D, Wasem J, Ambulante Krankenhausleistungen - ein Überblick, eine Trendanalyse und einige ordnungspolitische Anmerkungen. In: Klauber J, Geraedts M, Friedrich J et al., Hrsg. Krankenhaus-Report 2016: Ambulant im Krankenhaus. Stuttgart: Schattauer; 2016: 3-28

[21] Spitzenverbände der Krankenkassen und die Deutsche Krankenhausgesellschaft. Datenübermittlung nach § 301 Abs. 3 SGB V (Stand: Nachtrag vom 12.10.2018). 2018

[22] Brachmann M, Geppert R, Gröning M et al. Positionspapier der AG Ökonomie: Ökonomische Aspekte der klinischen Notfallversorgung. 2009

[23] Niehues C. Notfallversorgung in Deutschland: Analyse des Status quo und Empfehlungen für ein patientenorientiertes und effizientes Notfallmanagement. Stuttgart: Kohlhammer; 2012

[24] DIMDI. Basiswissen Kodieren: Eine kurze Einführung in die Anwendung von ICD-10-GM und OPS. Köln: Deutsches Institut für Medizinische Dokumentation und Information (DIMDI); 2010

[25] Schubert I, Ihle P, Köster I et al. Datengutachten für das Deutsche Institut für Medizinische Dokumentation und Information (DIMDI). Köln 2014

[26] von Stillfried D, Czihal T, Erhart M. Rolle der Krankenhäuser in der Notfallversorgung in Deutschland: Daten belegen massiven Reformbedarf. Zi-Paper 2017

[27] KBV Kassenärztliche Bundesvereinigung. Einheitlicher Bewertungsmaßstab (EBM) - Stand: 2. Quartal 2017

[28] Deutsche Krankenhausgesellschaft, GKV-Spitzenverband, Verband der privaten Krankenversicherung et al. Deutsche Kodierrichtlinien - Version 2018.

[29] GKV-Spitzenverband, Verband der Privaten Krankenversicherung, Deutsche Krankenhausgesellschaft. Vereinbarung zum Fallpauschalensystem für Krankenhäuser für das Jahr 2018. 2017

[30] GKV-Spitzenverband, Deutsche Krankenhausgesellschaft, Bundesvereinigung K. Ambulantes Operieren und sonstige stationsersetzende Eingriffe im Krankenhaus (AOP-Vertrag). 2014

[31] Deutsche Krankenhausgesellschaft. Vereinbarung mit den Trägern der gesetzlichen Unfallversicherung zur Datenübertragung von Abrechnungsdaten gemäß der Rahmenvereinbarung vom 5.12.2012. 2012 
[32] InEK. Daten nach § 21 KHEntgG - Version 2018 für das Datenjahr 2017 (Stand: Fortschreibung vom 22. Dezember 2017). 2017

[33] Statistisches Bundesamt. Fallpauschalenbezogene Krankenhausstatistik (DRG-Statistik) 2016. 2017

[34] KBV. Richtlinie der Kassenärztlichen Bundesvereinigung nach § 75 Absatz 7 SGB V zur Vergabe der Arzt-, Betriebsstätten- sowie der Praxisnetznummern 2017

[35] Huke T, Robra B-P. Notfallversorgung im stationären Sektor. In Klauber J, Geraedts M, Friedrich J et al., Hrsg. Krankenhaus-Report 2015: Strukturwandel. Stuttgart: Schattauer; 2015: 61-76

[36] Wahlster P, Czihal T, Gibis B et al. Sektorenübergreifende Entwicklungen in der Notfallversorgung - Eine umfassende Analyse ambulanter und stationärer Notfälle von 2009 bis 2015. Gesundheitswesen doi:10.1055/a-0820-3904

[37] Ahlbrandt J, Brammen D, Majeed RW et al. Balancing the need for big data and patient data privacy - an IT infrastructure for a decentralized emergency care research database. Stud Health Technol Inform 2014; 205: 750-754

[38] Kulla M, Brammen D, Greiner F et al. Vom Protokoll zum Register Entwicklungen für ein bundesweites Qualitätsmanagement in deutschen Notaufnahmen. DIVI 2016; 7: 12-20. doi:10.3238/ DIVI.2016.0012-0020

[39] Kulla M, Baacke M, Schöpke T et al. Kerndatensatz „Notaufnahme“ der DIVI. Notfall Rettungsmed 2014; 17: 671-681. doi:10.1007/ s10049-014-1860-9
[40] Schreyögg ], Bäuml M, Krämer ] et al. Forschungsauftrag zur Mengenentwicklung nach § 17b Abs. 9 KHG 2014

[41] Krämer J, Schreyögg J, Busse R. Classification of hospital admissions into emergency and elective care: a machine learning approach. Health care management science 2019; 22: 85-105. doi:10.1007| s10729-017-9423-5

[42] Swart E, Stallmann C, Powietzka J et al. Datenlinkage von Primär- und Sekundärdaten. Bundesgesundheitsblatt - Gesundheitsforschung Gesundheitsschutz 2014; 57: 180-187. doi:10.1007/s00103-0131892-1

[43] GKV-Spitzenverband. Neustrukturierung der Notfallversorgung - Positionspapier des GKV-Spitzenverbandes. 2017

[44] Meier F, Bauer K, Schöffski O et al. Zur Ökonomie ambulanter Notaufnahmepatienten. Notfall Rettungsmed 2015; 19: 33-40. doi:10.1007/s10049-015-0054-4

[45] Greiner F, Brammen D, Kulla M et al. Standardisierte Erhebung von Vorstellungsgründen in der Notaufnahme. Medizinische Klinik - Intensivmedizin und Notfallmedizin 2018; 113: 115-123. doi:10.1007| s00063-017-0286-9

[46] Brammen D, Greiner F, Dormann $\mathrm{H}$ et al. Lessons learned in applying the International Society for Pharmacoeconomics and Outcomes Research methodology to translating Canadian Emergency Department Information System Presenting Complaints List into German. Eur J Emerg Med 2018; 25: 295-299. doi:10.1097/mej.0000000000000450 